\title{
SOLAR SOFT X-RAYS AND SOLAR ACTIVITY
}

\author{
II. Soft X-Ray Emission during Solar Flares
}

\author{
ROGER J. THOMAS* \\ Dept. of Astronomy and the Mc Math-Hulbert Observatory, \\ The University of Michigan, Ann Arbor, Michigan and Laboratory for Solar Physics, NASA/Goddard \\ Space Flight Center, Greenbelt, Maryland \\ and \\ RICHARD G. TESKE \\ Dept. of Astronomy and the McMath-Hulbert Observatory, \\ The University of Michigan, Ann Arbor, Michigan
}

(Received 22 September, 1970)

\begin{abstract}
Flare-associated soft X-ray bursts (8-12 $\AA$ ) are examined for 283 events observed by OSO-III. These bursts are shown to be predominantly thermal in nature. Their time-profiles are roughly similar to those of the associated $\mathrm{H} \alpha$ flares, although the $\mathrm{X}$-ray burst begins about two minutes earlier, on the average. The strength of the soft X-ray burst is directly related to the area and brilliance of the flare, the age and 'flare-richness' of the associated plage, and the general level of solar activity at the time of the burst. The peak enhancements in the soft X-ray and $\mathrm{H} \alpha$ emission rates during flares are of the same order of magnitude, as are the total flare energies radiated at these wavelengths. We estimate that soft X-radiation accounts for up to $10 \%$ of a flare's total electromagnetic emission.
\end{abstract}

\section{Introduction}

The University of Michigan operated a solar soft X-ray ion chamber photometer on board the OSO-III satellite. Between 9 March, 1967 and 28 June, 1968, records of solar X-radiation between $8-12 \AA$ of exceptional quality were obtained. The characteristics of the instrument, its operation and its calibration have been discussed extensively elsewhere (Teske, 1969; Thomas, 1970a).

We have previously reported an analysis of the association of soft X-ray bursts with solar flares (Teske and Thomas, 1969; hereafter called Paper I). In the present communication we will study in more detail some aspects of the flare/soft X-ray relationship to confirm, revise and extend the results of Paper I. In particular we wish to examine in a quantitative manner the statistical relations that exist between the soft $\mathrm{X}$-ray and visible $\mathrm{H} \alpha$ radiation from flares. One of us (Thomas, 1970a) has shown that the flux scale of the Michigan OSO-III instrument is reliable, thus permitting confident evaluation of quantitative results.

For this study, we compiled a new catalog of well-confirmed solar flares of importance $\geqslant 1$ (called Catalog II), but based upon somewhat different criteria than those discussed in Paper I. The present criteria were designed to eliminate the clear

* NRC/NAS Resident Research Associate. 
bias found in Catalog I towards events occurring in the UT interval 0600-1400 hr. The new criteria require, among other things, that the majority of the optical stations monitoring the Sun at the time of a flare maximum actually reported the flare, and that the majority of those reporting a flare agreed that its importance was $\geqslant 1$. At least one reporting station had to be photographic or cinematographic. The flare importance assigned by the Quarterly Bulletin on Solar Activity (QB) was used by us. Flares overlapping in time with other flares or subflares were eliminated so that only well-confirmed, single events were studied. Our final Catalog II contained 283 events for which at least partial X-ray coverage exists in the time interval 9 March, 1967 through 31 March, 1968. The catalog represents a significantly different sample of flares than that upon which Paper I was based, although many events are common to both lists.

\section{Relative Timing of $\mathrm{H} \alpha$ and $\mathrm{X}$-Ray Enhancements in Flares}

The timing of X-ray bursts relative to the visible flare is of interest because of the indication that a soft $\mathrm{X}$-ray enhancement is often the first manifestation of a solar flare (Donnelly, 1968; and Paper I). The time-relations between $\mathrm{H} \alpha$ flares and X-ray bursts have already been examined in Paper I using our first catalog. However, because of its importance, the question of early X-rays has been re-analyzed here with the more extensive list of events in Catalog II. The results re-emphasize that a weak soft X-ray enhancement often precedes the $\mathrm{H} \alpha$ brightening of a flare, but not always.

\section{A. STARTING TIMES}

The starting time of an $\mathrm{H} \alpha$ flare has been taken as the earliest start-time reported by a cinematographic or photographic flare patrol station, as before. The bursts were divided into groups depending upon whether they started when our detector was operating in its high sensitivity mode or low sensitivity mode, and were further divided as to whether the bursts were associated with flares within $60^{\circ}$ heliocentric of the disk center or were associated with flares nearer the limb (called 'center' and 'limb' events, respectively). Table I shows the results of this study, which included a total of 173 events. The average differences between starting times are given in minutes, and negative values mean that the $\mathrm{X}$-ray burst starts earlier than the $\mathrm{H} \alpha$ flare. The numbers in parentheses indicate how many events entered into each average. Also listed is a

TABLE I

Mean differences between $\mathrm{H} \alpha$ and soft X-ray starting times (All time differences are in minutes)

\begin{tabular}{lllr} 
& \multicolumn{1}{c}{ Center } & Limb & \multicolumn{1}{c}{$P$} \\
\hline Low sensitivity & $+0.2(88)$ & $+0.1(39)$ & $8 \%$ \\
High sensitivity & $-3.4(33)$ & $-4.8(13)$ & $57 \%$ \\
$P:$ & $>99 \%$ & $97 \%$ & \\
\hline
\end{tabular}


parameter $P$ (calculated by Student's $t$-test) which shows the significance of the observed difference between the two sample means. Although the soft X-ray burst begins early relative to the $\mathrm{H} \alpha$ flare, on the average, there is a wide variation in the individual values. The range found here extends from X-rays starting early by $28 \mathrm{~min}$ to $\mathrm{X}$-rays starting late by $12 \mathrm{~min}$. Even when we restrict our attention to high sensitivity operation only, about $30 \%$ of the X-ray bursts started after the reported start-time of the associated $\mathrm{H} \alpha$ flare. This great dispersion of values is undoubtedly responsible for the conflicting results of other timing studies (cf. Landini et al., 1965; Falciani et al., 1968). The results of Table I confirm those in Paper I, Table II, but the larger sample of flares here makes the current result the more reliable.

\section{B. THE X-RAY PRECURSOR}

Although in Paper I we suggested designating the early rise of X-rays the 'predecessor' to distinguish it from the $\mathrm{cm}-\lambda$ precursor which usually begins at the same time as the $\mathrm{H} \alpha$ flare, the term precursor has now generally been applied by other investigators to the early X-rays.

Counterparts to the soft X-ray precursor have been described for $1-1.5 \AA$ bursts (Hudson et al., 1969) and for 3-4 $\AA$ bursts (Culhane and Phillips, 1970). However, the analysis leading to the identification of a precursor contains two known sources of systematic error, at least in the case of the soft X-rays.

The first source for error is in the designation of the $\mathrm{H} \alpha$ flare's starting time, which was taken by us to be the earliest reported cinematographic or photographic starting time. Cinematography offers a good time resolution, but even so, filtroheliograms are normally made only once each $30 \mathrm{sec}$. Further, the observed flare start is given as the time of the first frame on which a visually detectable brightening occurs. This combination of practices results in reported starting times which are consistently later than the 'true' beginning of an event. Angle (1968) suggests from a photometric study that the rapid-rise phase of the $\mathrm{H} \alpha$ flare may begin up to $1-1 \frac{1}{2}$ min on the average before it is defined from a visual inspection of the film.

The second source of systematic error is the existence of soft X-ray fluctuations which are not related to any reported optical events (Gregory and Kreplin, 1967; Teske, 1967). While care was taken in forming Catalog II to eliminate overlapping reported optical events, the background fluctuations may have contaminated the $\mathrm{X}$-ray data.

In order to estimate the influence of the background fluctuations on the mean starttime relations of Table $I$, the frequency and duration of non-flare-associated X-ray variations were investigated for the period of 10 March, 1967 to 18 April, 1967. Since the soft X-ray burst precursor usually appears only in data obtained during high-sensitivity operation of the detector, only high-sensitivity data were examined in detail. The total time-interval examined was $7371 \mathrm{~min}$, called $C$ hereafter. By determining the fraction of the test interval $C$ affected by the background fluctuations, one can calculate the amount of the average early rise given in Table I which is due to this effect. 
If $N(d)$ is the number of such fluctuations with duration $d$ occurring during the test interval, the fraction of this interval in which the burst starting times would be mis-identified by $t$ or more minutes is given by:

$$
X(t)=\sum_{d=t}^{\infty}(d-t) N(d) / C
$$

(Only integral values of $d$ and $t$ were considered.) The fraction of the interval in which starting times would be assigned exactly $t$ minutes too early is then:

$$
Y(t)=X(t)-X(t+1) .
$$

Finally, the effect of background fluctuations accounts for $Z$ minutes in the average early rise-time of bursts occurring at random during the test interval, where $Z$ is given by:

$$
Z=\sum_{t=0}^{\infty} t Y(t)
$$

The frequency distribution of $N(d)$ for the time-interval studied here is shown in Figure 1 and results in a value of $Z=1.2 \mathrm{~min}$. Since the interval studied does not appear atypical, we assume that this value of $Z$ can be applied to the relative start-

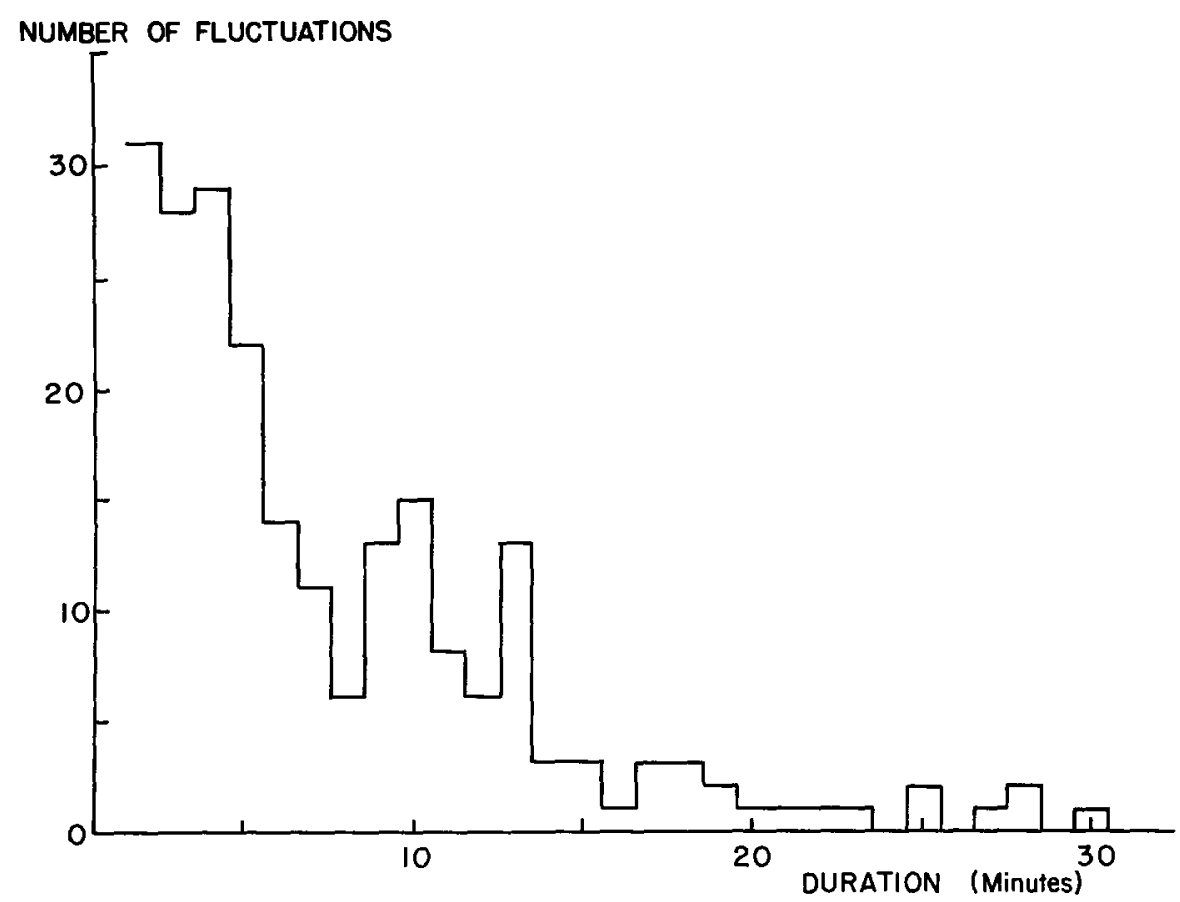

Fig. 1. Frequency distribution of 8-12 $\AA$ background fluctuations as a function of duration. Such fluctuations are soft X-ray enhancements which do not accompany any reported optical activity on the Sun. This distribution refers to the $7371 \mathrm{~min}$ of high-sensitivity data recorded by the Michigan detector between 10 March, 1967 and 18 April, 1967. 
time relations found for all Catalog II events. Thus, taking $1.2 \mathrm{~min}$ for the error caused by the background variations and $1.5 \mathrm{~min}$ for the error due to the method of assigning $\mathrm{H} \alpha$ flare starting times, we find that a soft $\mathrm{X}$-ray burst begins on the average 1.1 min before its associated flare, if center and limb events are considered together in Table $\mathbf{I}$.

The above analysis of the effect of the background fluctuations upon relative starting times of X-ray bursts and $H \alpha$ flares assumes that the background variations are not physically related to the flare itself. Culhane (personal communication) has pointed out another possibility, namely that the background variations are manifestations of a flare triggering mechanism that is not always successful. By this interpretation, all observed precursors are valid components of the X-ray burst and the average early rise of soft X-rays relative to the $\mathrm{H} \alpha$ flare becomes $2.3 \mathrm{~min}$. The hypothesis also implies that two presumably independent conditions must obtain simultaneously in order for the total flare phenomenon to be initiated: first, the existence of the triggering agent, which may always give rise to a soft X-ray enhancement, and second, the metastable high-energy configuration of the potential flare source. Thus, if this suggestion is correct, a careful examination of a plage region during flare-producing and non-flare-producing precursors might indicate the active center configuration necessary for a flare event to occur. Such a study merits future consideration.

\section{MAXIMUM AND ENDING TIMES}

Timing relations for the maximum and ending phases of $\mathrm{H} \alpha$ flares relative to their associated soft X-ray bursts have been determined for events in Catalog II. The results are shown in Table II, where the terms 'center' and 'limb', and the parameter $P$, are defined as before. Here the positive values mean that, on the average, the soft X-ray maximum follows the $\mathrm{H} \alpha$ intensity maximum, and that the soft $\mathrm{X}$-rays outlast the visible flare. These data qualitatively confirm the results of Paper I, Table III, but represent a revision of the lag times given therein. Further, the sense of a possible center-to-limb variation is reversed.

Unlike the hard X-rays, it appears to be quite rare for the soft X-ray burst maximum to precede the peak $\mathrm{H} \alpha$ intensity of a flare: this occurred in only $5 \%$ of the cases studied here.

The time of ending of an X-ray burst is often difficult to identify with confidence, as is the identification of the time of ending of the $\mathrm{H} \alpha$ flare itself (Dodson and

TABLE II

Mean differences between $\mathrm{H} \alpha$ and soft $\mathrm{X}$-ray maximum and ending times

(All time differences are in minutes)

\begin{tabular}{llll}
\hline & Center & Limb & $P$ \\
\hline Times of maximum & $+3.6(160)$ & $+2.3(71)$ & $96 \%$ \\
Times of end & $+13.9(75)$ & $+9.6(31)$ & $80 \%$
\end{tabular}


Hedeman, 1964). Thus, the relative time-relation for event cessation given in Table 11 must be viewed as just an approximation. The scatter among the different values is very large, ranging from the $\mathrm{X}$-rays ending early by $38 \mathrm{~min}$ to the $\mathrm{X}$-rays ending late by $58 \mathrm{~min}$, relative to the $\mathrm{H} \alpha$ flare. Because of the uncertainties, the center-to-limb difference for the relative ending times cannot be considered as established. On the other hand, the difference between relative times of maxima for flares near disk center and near the limb, although small, does appear to be significant (Table II).

\section{DURATION OF SOFT X-RAY BURSTS}

It is well known that the total durations of $\mathbf{H} \alpha$ flares depend upon their importance on the average (e.g., Waldmeier and Bachmann, 1959; Smith, 1962). Unfortunately, Catalog II is not extensive enough to permit a similar study for the X-ray bursts associated with these flares. Of the 60 bursts in the catalog which had observed beginning and ending times, 52 accompanied importance 1 flares. For these, the median burst duration is $33 \mathrm{~min}$, somewhat longer than the median duration of $24 \mathrm{~min}$ found for importance $1 \mathrm{H} \alpha$ flares in general (Smith, 1962). The typical 'importance 1' $\mathrm{X}$-ray burst is probably of even greater extent, however, because the above procedure systematically discriminates against longer duration events. The end-times for longenduring bursts are much less likely to be identified since they have more chance of being contaminated by other events or lasting into the unobserved period during satellite-night. Therefore, we conclude that the soft X-rays accompanying flares have a greater duration than their optical counterparts on the average, a result which agrees with the observations of others (Acton et al., 1963; Lindsay, 1964; Paolini et al., 1968).

\section{E. RISE-TIME CHARACTERISTICS OF SOFT X-RAY BURSTS}

Solar microwave bursts usually fall into one of two categories, normally called gradual rise and fall (GRF) and impulsive bursts (Covington and Harvey, 1958). This classification also applies to hard X-ray events (Kane, 1969), but the situation is not so clear for X-ray bursts at longer wavelengths. Some authors claim that such a distinction can be clearly made for soft X-ray events (Chambe and Sain, 1969), some find only slight evidence for it (Culhane et al., 1963; Culhane and Phillips, 1970), while others feel no such distinction exists (Drake, 1969). The resolution of this problem is of great importance because of the insight it would give into the physical characteristics of the solar X-ray emitting region. In the case of the microwave events, for example, it is generally agreed that the GRF bursts are thermal in nature, while impulsive bursts are due to non-thermal processes (e.g., Takakura and Kai, 1966; Holt and Cline, 1968).

Two semi-related parameters can be used to distinguish gradual events from impulsive ones: the time between the start and maximum of a burst $\Delta t$, and the mean rate of flux enhancement $\Delta E / \Delta t$. A total of 128 soft X-ray bursts in Catalog II were sufficiently well observed so that these parameters could be determined for them. The frequency distributions of events with various values of these parameters are 


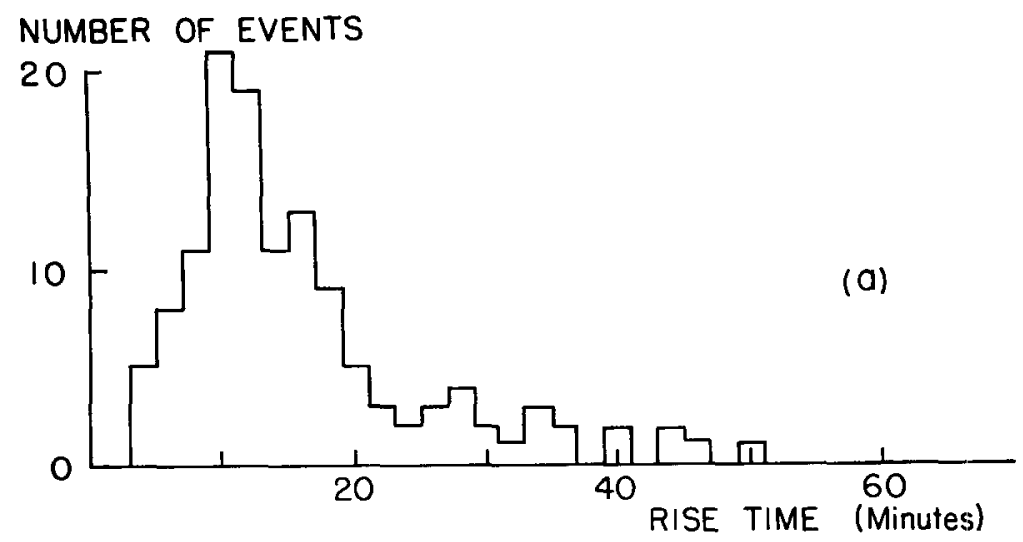

NUMBER OF EVENTS

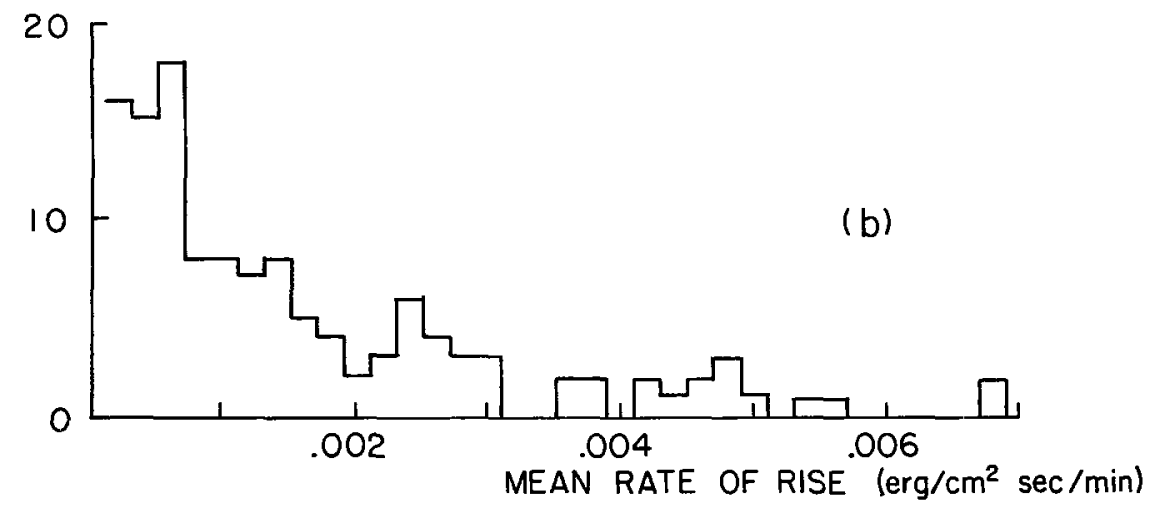

Fig. 2. Frequency distributions of Catalog II bursts with (a) rise-time and (b) mean rate of rise. Neither distribution is bi-modal in character.

depicted in Figure 2. Neither distribution shows any evidence for two distinct burst types, which suggests that all soft X-ray events can be considered of thermal origin. The time-development is much too gradual on the average to assume that they are all nonthermal. Another attempt to identify impulsive bursts in Catalog II is shown as Figure 3 . This plot of the soft $\mathrm{X}$-ray amplitude $\Delta E$ versus the rise-time $\Delta t$ is essentially the same method originally used to distinguish the two types of microwave bursts (Covington, 1959). Again, no clear grouping occurs for the X-ray events considered here.

The list of X-ray bursts used in the present study is a very select one, consisting only of those which accompany well-verified $\mathrm{H} \alpha$ flares. Thus, it is conceivable that the result found here does not apply to other soft X-ray bursts. But this does not seem to be the case, since it best agrees with Drake's (1969) investigation, the most extensive yet attempted, which includes over 2000 events observed during the period 2 July, 1966 to 18 September, 1968. De Jager (1965), who first suggested that X-ray bursts be divided into 'quasi-thermal' and 'nonthermal' classes, also felt the majority 


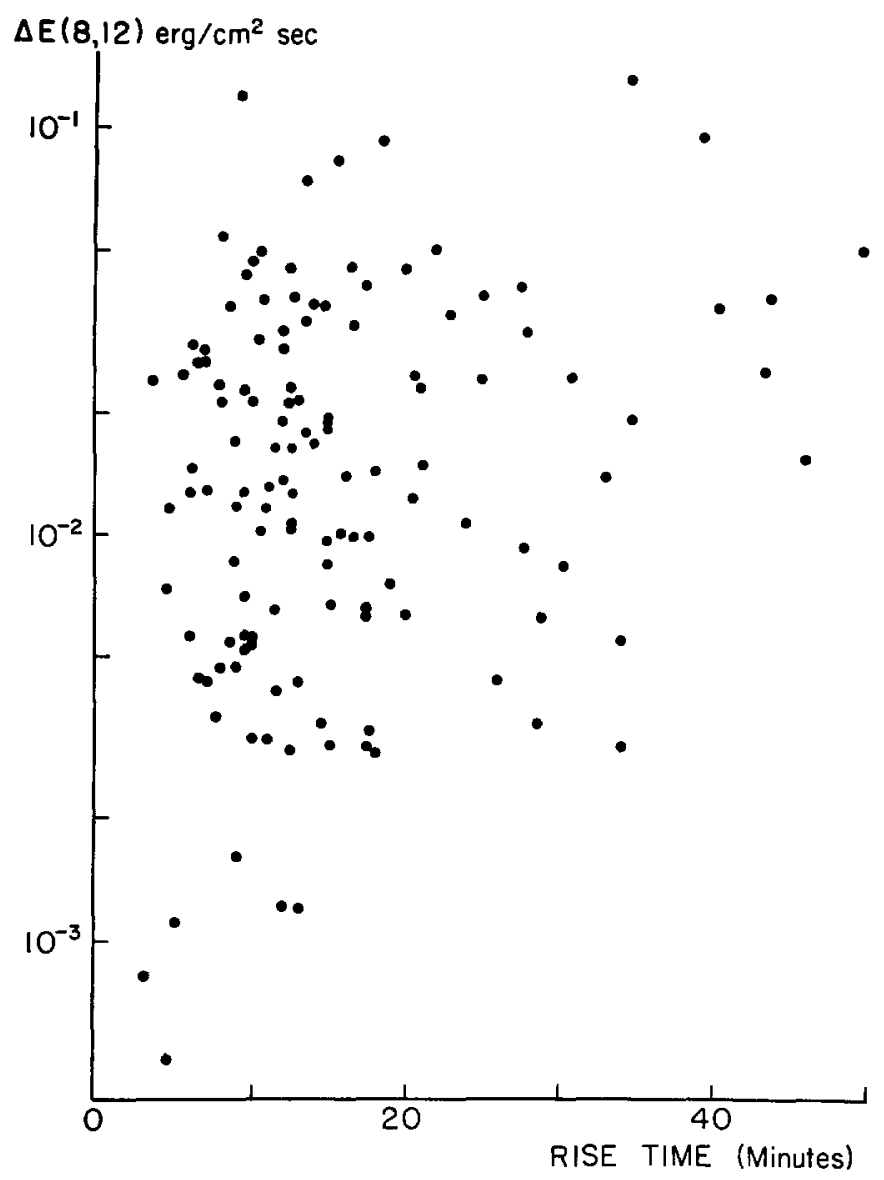

Fig. 3. Scatter diagram of X-ray burst amplitude $\Delta E(8,12)$ versus rise-time for events of Catalog II. No evidence appears for distinct classes of soft X-ray bursts.

of bursts observed at wavelengths longer than $1 \AA$ should fall in the former category. Other theoretical investigations led to similar conclusions (Kawabata, 1963, 1966; Kundu, 1964; Culhane and Phillips, 1970).

\section{Soft X-Ray Bursts Compared with Other Flare Phenomena}

The ion chamber currents measured by our instrument were converted to soft X-ray fluxes under the assumption that the $\mathrm{X}$-ray spectrum is constantly like that of a black body at $2 \times 10^{6} \mathrm{~K}$. One of us (Thomas, 1970a) has investigated the effects of different spectral distributions on the quoted flux values, using various theoretical spectra that may reasonably be expected to be observed, and by making comparisons with simultaneous observations made by other instruments. In the worst case, the relative error of the quoted fluxes $-E(8,12) \mathrm{erg} \mathrm{cm}^{-2} \mathrm{sec}^{-1}-$ is in the neighborhood of $30 \%$. 
(However, the quoted absolute values may be too large, perhaps by as much as a factor of two or three.) Thus spectrum changes during the course of a flare will not affect the relative quantitative comparisons to be made below.

Eight of the flares listed in Catalog II produced X-ray bursts which saturated the Michigan detector. It was possible to estimate the amplitude of these bursts by comparing our data with that collected by the University of Iowa's 2-12 $\AA$ detector on board Explorer-35 (Drake, personal communication). Intercomparison of the amplitudes of X-ray bursts observed in common by the Michigan and Iowa experiments gave the relation portrayed in Figure 4, an excellent relation holding over

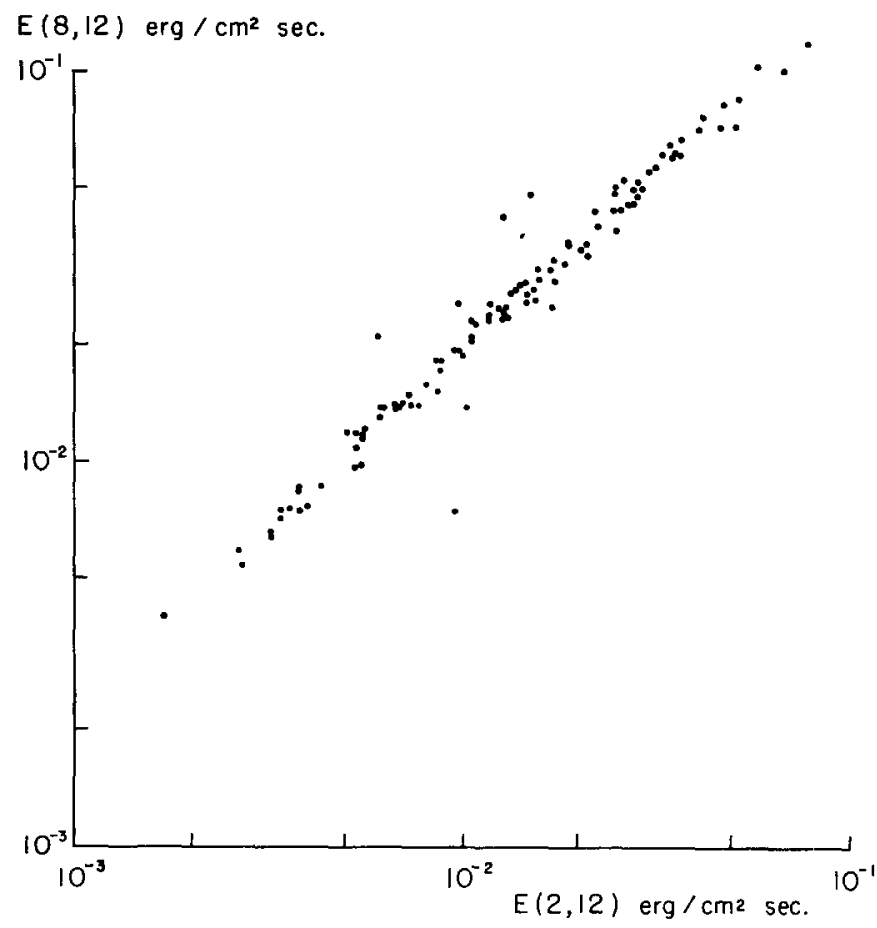

Fig. 4. Comparison of the peak $2-12 \AA$ and $8-12 \AA$ fluxes for $\mathrm{X}$-ray bursts associated with $\mathrm{H} a$ flares observed in common by a University of Iowa experiment aboard Explorer-35 and a University of Michigan experiment aboard OSO-III, respectively. The six points which do not lie directly on the nominal relation were marked as uncertain by one or both investigators.

nearly two orders of magnitude. Assuming that an extrapolation of this relation was valid, and using the X-ray burst amplitude observed by Iowa for those flares that had saturated the Michigan instrument, we estimated the 8-12 $\AA$ X-ray amplitude that should have been observed by Michigan.

\section{A. FREQUENCY OF OCCURRENCE OF X-RAY BURSTS WITH FLARES}

In Paper I we found that, of 103 flares for which X-ray observations were being made 
near the time of flare maximum, only one appeared to be unaccompanied by soft $\mathrm{X}$-rays between 8 and $12 \AA$. Among the 283 flares of Catalog II, we have found only one (importance 1f) for which the X-rays were not detected by our instrument. At the time of that flare, the instrument was operating in its 'flare mode' of low sensitivity (cf. Teske and Thomas, 1969); thus the upper limit to the accompanying X-ray enhancement was $0.0095 \mathrm{erg} \mathrm{cm}^{-2} \mathrm{sec}^{-1}$ at the Earth. We have concluded that essentially all flares of importance $\geqslant 1$ give rise to soft $\mathrm{X}$-radiation.

\section{B. SOFT X-RAY AMPLITUDE COMPARED WITH FLARE IMPORTANCE}

As in Paper I, the present study implies that soft X-ray burst amplitudes are related to the importance of the associated flare, but only in a statistical manner. This can be shown by grouping together all X-ray bursts in Catalog II which occurred during flares of each importance class, and calculating a mean $X$-ray amplitude, $\overline{\Delta E}$, for each group. Table III lists the values which result, along with the probable error (p.e.) of each mean, the number of events $(N)$ included, and the range of $\Delta E$ values observed for each group. The table shows that the average amplitude is directly related not only to the flare's area but also to its brightness. Note, however, that there is a very large range of amplitude within each importance class.

TABLE III

Mean soft X-ray amplitudes for flare-associated bursts (all flux values are in erg $\mathrm{cm}^{-2} \mathrm{sec}^{-1}$ )

\begin{tabular}{|c|c|c|c|c|c|}
\hline $\begin{array}{l}\text { Flare } \\
\text { importance }\end{array}$ & $\overline{\Delta E}$ & p.e. & $N$ & $\begin{array}{l}\text { Minimum } \\
\Delta E\end{array}$ & $\begin{array}{l}\text { Maximum } \\
\Delta E\end{array}$ \\
\hline If & 0.0052 & 0.0010 & 11 & 0.0000 & 0.0149 \\
\hline $1 \mathrm{n}$ & 0.0183 & 0.0014 & 138 & 0.0005 & 0.2163 \\
\hline $1 b$ & 0.0345 & 0.0028 & 50 & 0.0043 & 0.1912 \\
\hline $2 f$ & 0.0248 & - & 1 & - & - \\
\hline $2 n$ & 0.0423 & 0.0127 & 13 & 0.0031 & 0.2635 \\
\hline $2 b$ & 0.0970 & 0.0209 & 11 & 0.0390 & 0.3909 \\
\hline $3 n$ & 0.1328 & - & 1 & - & - \\
\hline $3 b$ & 0.8060 & - & 1 & - & - \\
\hline
\end{tabular}

In order to present the relationship in a quantitative form, the average burst amplitudes were converted into the corresponding emission-rate enhancements at the Sun, $\Delta S(8,12)$, under the assumption that the soft X-radiation is isotropic into $4 \pi$ steradians. The resulting values of $\Delta S(8,12)$ can then be directly compared to the appropriate $\mathrm{H} \alpha$ emission-rate enhancements at flare maximum, $\Delta S(\mathrm{H} \alpha)$, as found by Thomas (1970b). Such a comparison is shown in Figure 5, which includes values for subflares that were measured during the construction of an earlier catalog. The latter naturally should not be given as much weight as those derived from the flares in Catalog II, but are included to point out that the relation found here may also extend to subflares. 


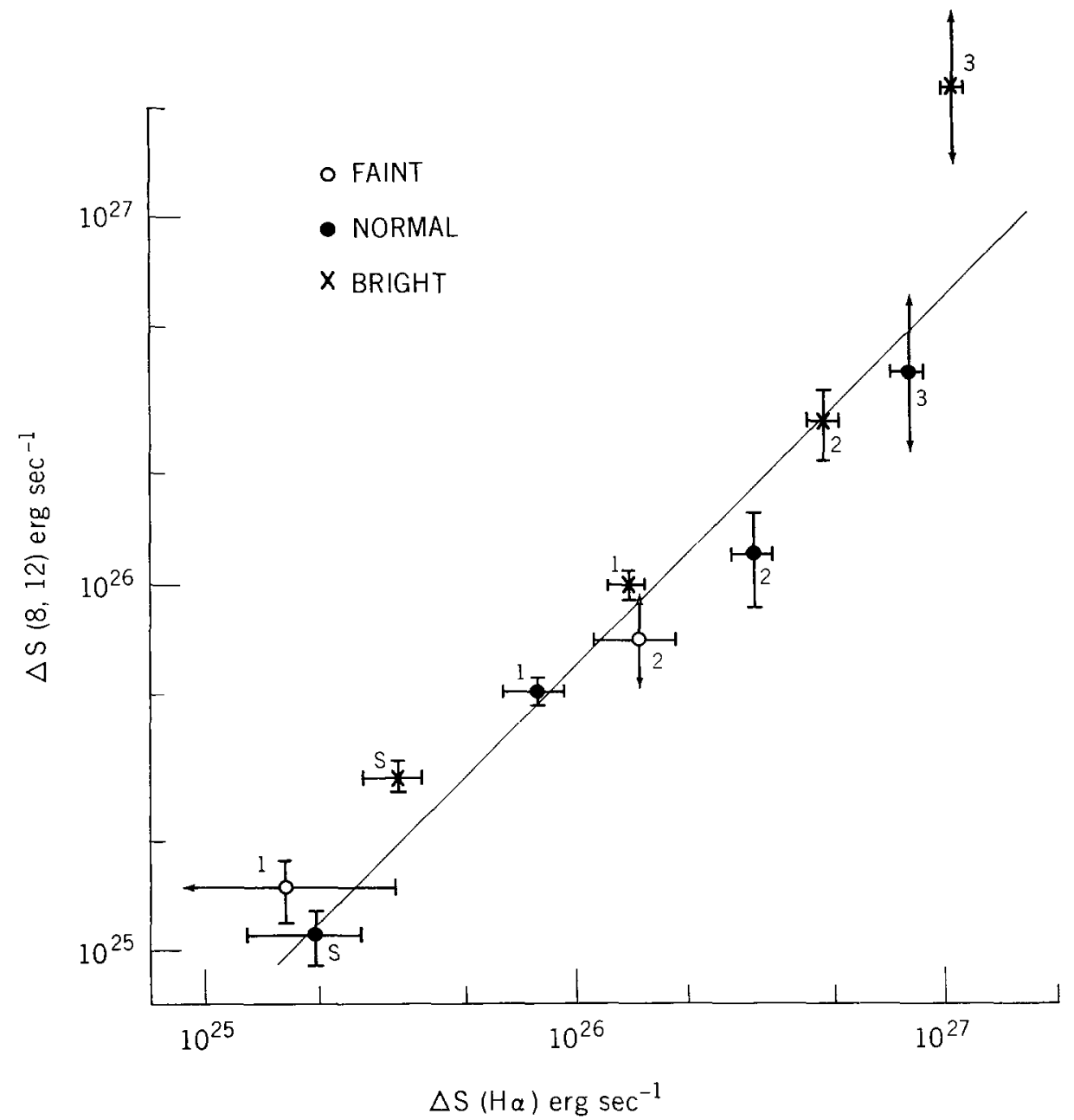

Fig. 5. Relation between peak emission rates of $8-12 \AA$ and $\mathrm{H} a$ radiation for various flare importances. Error bars refer to the probable errors involved, except that no error can be assigned to $\Delta S(8,12)$ associated with $2 \mathrm{f}, 3 \mathrm{n}$, or $3 \mathrm{~b}$ flares, since only one example of each of these bursts has been measured. The straight line indicates the best fit by a linear relationship (i.e., unity slope).

The straight line in Figure 5 indicates the best linear fit (unity slope) to the plotted points, and shows that there is an excellent statistical relationship between the peak rates of $\mathrm{H} \alpha$ and soft $\mathrm{X}$-ray enhancements which is well represented by a direct proportionality. The best-fit relationship gives:

$$
\Delta S(8,12)=0.6 \Delta S(\mathrm{H} \alpha) \text {. }
$$

However, note that the above relationship applies to emission rates at the maximum of the $\mathrm{H} \alpha$ flare and at the maximum of the X-ray burst. As shown in Section $2 \mathrm{C}$, these maxima occur in general at different times. 
There is some evidence that European observatories as a whole tend to rank flares on a slightly higher importance scale than do observatories in other parts of the world (Dodson and Hedeman, 1960; Warwick, 1965). To investigate this question using the X-ray data of Catalog II, the mean amplitudes were determined for all bursts accompanying importance 1n flares whose maxima occurred during 'European', 'American', and 'Asian' observing hours: defined here as 0600-1359, 1400-2259, and 2300-1559 UT, respectively. Table IV shows that such bursts do have lower average

TABLE IV

Mean soft X-ray amplitude for importance $1 \mathrm{n}$ flares versus universal time

\begin{tabular}{lll}
\hline $\begin{array}{l}\text { Time of } \\
\text { maximum (UT) }\end{array}$ & Observers & $\begin{array}{l}\overline{A E}_{1 \mathrm{n}}(8,12) \\
\mathrm{erg} \mathrm{cm} \mathrm{sec}^{-1}\end{array}$ \\
\hline $2300-0559$ & 'Asian' & $0.0238 \pm 0.0034$ p.e. \\
$0600-1359$ & 'European' & $0.0147 \pm 0.0008$ \\
$1400-2259$ & 'American' & $0.0211 \pm 0.0040$ \\
\hline
\end{tabular}

amplitudes when associated with reported flares that can be attributed roughly to European observers. This is exactly the result expected if these observers did indeed rate flares as importance $1 \mathrm{n}$ which would have been assigned somewhat lower rankings elsewhere. This is not meant to imply in any way, of course, that the reports from some flare-patrol stations are correct while others are not. Rather, it merely substantiates certain claims that world-wide ratings of solar flare importances are not made on a uniform basis at the present time. This fact undoubtedly accounts for a part of the burst amplitude variations described earlier, and points out one advantage of studying flare characteristics by means of statistical investigations which tend to average out such effects.

\section{SOFT X-RAY AMPLITUDE COMPARED WITH DISK LOCATION OF FLARE}

The average amplitude of X-ray bursts accompanying importance $1 \mathrm{n}$ flares (the most prevalent in our sample) was also considered as a function of the position of the burst on the solar disk. This was done in two ways: (a) by dividing the Sun into its various hemispheres and quadrants, and (b) by comparing bursts that accompanied flares near the limb with those that occurred toward the center of the disk. The division into 'center' and 'limb' events was the same as that described in Section 2A. Table V gives the results of these studies and shows that there are no strong statistical differences among the categories tested. The same result has been reported for similar studies using data from the effects of Sudden Ionospheric Disturbances (Giovanelli, 1938; Warwick, 1963; Reid, 1969).

However, there is some suggestion of a moderate center-to-limb effect since the most significant difference in Table $V$ is between the center and limb bursts. Student's $t$-test implies that this difference has only a $13 \%$ probability of being due purely to 
TABLE V

Mean soft X-ray amplitude for importance in flares versus location on the disk

\begin{tabular}{|c|c|}
\hline Location & 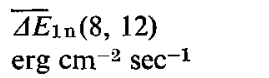 \\
\hline $\begin{array}{l}\text { Northern hemisphere } \\
\text { Southern hemisphere }\end{array}$ & $\begin{array}{l}0.0191 \pm 0.0015 \text { p.e. } \\
0.0150 \quad 0.0015\end{array}$ \\
\hline $\begin{array}{l}\text { Eastern hemisphere } \\
\text { Western hemisphere }\end{array}$ & $\begin{array}{l}0.0174 \pm 0.0016 \text { p.e. } \\
0.01820 .0015\end{array}$ \\
\hline $\begin{array}{l}\text { N-E quadrant } \\
\text { N-W quadrant } \\
\text { S-E quadrant } \\
\text { S-W quadrant }\end{array}$ & $\begin{array}{ll}0.0186 \pm 0.0022 \text { p.e. } \\
0.0197 & 0.0019 \\
0.0143 & 0.0013 \\
0.0156 & 0.0026\end{array}$ \\
\hline $\begin{array}{l}\text { Center }(\mu \geqslant 0.5) \\
\text { Limb }(\mu<0.5)\end{array}$ & $\begin{array}{l}0.0161 \pm 0.0012 \text { p.e. } \\
0.02180 .0024\end{array}$ \\
\hline
\end{tabular}

TABLE VI

Mean soft X-ray amplitude for importance $1 \mathrm{n}$ flares versus disk-center distance

\begin{tabular}{lll}
\hline$R$ & $\overline{\Delta E}_{1 \mathrm{n}}(8,12)$ \\
Solar radii & \multicolumn{2}{l}{$\mathrm{erg} \mathrm{cm}^{-2} \mathrm{sec}^{-1}$} \\
\hline $0.000-0.400$ & & $0.0139 \pm 0.0027$ \\
$0.401-0.600$ & 0.0146 & 0.0014 \\
$0.601-0.800$ & 0.0173 & 0.0024 \\
$0.801-0.960$ & 0.0213 & 0.0026 \\
$0.961-1.000$ & 0.0249 & 0.0061 \\
\hline
\end{tabular}

chance. The trend is shown in more detail in Table VI, where the data are subdivided into smaller intervals of $R$, the distance of the flare from the center of the disk in units of solar radii. The intervals of $R$ used here were chosen to include roughly equal numbers of events. Note that the tabulated values increase monotonically as the limb is approached. This result is consistent with the study by Dodson et al. (1956), which showed that SID's were larger on the average for limb flares of a given importance. Such an effect is most likely due to the reduced visibility of $\mathrm{H} \alpha$ flares near the limb (Dodson and Hedeman, 1964) which leads to the general tendency for their importance to be underestimated (Sawyer, 1967).

D. SOFT X-RAY AMPLITUDE RELATED TO GENERAL LEVEL OF SOLAR ACTIVITY

The nonburst component of the soft X-ray flux is a good index of the general solar activity level (e.g. Teske, 1969; Wende, 1969). Whenever the flux exceeded $0.0044 \mathrm{erg} \mathrm{cm}^{-2} \mathrm{sec}^{-1}$, the Michigan experiment automatically switched into its low sensitivity mode of operation. Thus, X-ray bursts that began when the detector was 
in its low sensitivity mode can be considered as having occurred during a time of 'high' general activity. Table VII shows that such X-ray bursts have a significantly higher amplitude on the average than those starting in the detector's high sensitivity mode, since Student's $t$-test implies that the observed difference between these two categories has less than $1 \%$ probability of being due to chance. We conclude that X-ray bursts accompanying importance $1 \mathrm{n}$ flares tend to have greater amplitudes on those days when the general level of solar activity is high.

TABLE VII

Mean soft X-ray amplitude for importance in flares versus initial detector mode

\begin{tabular}{ll}
$\begin{array}{l}\text { Initial operating mode } \\
\text { of detector }\end{array}$ & $\begin{array}{l}\overline{A E}_{1 \mathrm{n}}(8,12) \\
\mathrm{erg} \mathrm{cm}^{-2} \mathrm{sec}^{-1}\end{array}$ \\
\hline $\begin{array}{l}\text { High sensitivity } \\
\text { Low sensitivity }\end{array}$ & $\begin{array}{l}0.0094 \pm 0.0009 \mathrm{p} . e . \\
0.0212 \pm 0.0015\end{array}$
\end{tabular}

The same result is derived from Table VIII, which gives the correlation coefficients for soft X-ray enhancements during importance 1 n flares versus the background flux $E_{B}(8,12)$ and the Zürich sunspot number $R_{Z}$. Specifically, the latter coefficient relates the monthly mean of the final Zürich sunspot numbers to the mean X-ray enhancement for all importance $1 \mathrm{n}$ flares in Catalog II which occurred during the month in question. Values of $R_{Z}$ were taken from the ESSA Solar-Geophysical Data Bulletin (SGDB). In addition, the table indicates the probable significance $P$ for each of these two coefficients.

\section{TABLE VIII}

Correlation of soft X-ray amplitude for importance $1 \mathrm{n}$ flares with solar-activity indices

\begin{tabular}{llr}
$\begin{array}{l}\text { Index of general solar- } \\
\text { activity level }\end{array}$ & $\begin{array}{l}\text { Correlation } \\
\text { coefficient }\end{array}$ & $P$ \\
\hline $\begin{array}{l}E_{B}(8,12) \\
R_{Z}\end{array}$ & +0.41 & $>99 \%$ \\
+0.60 & $96 \%$
\end{tabular}

The relationship indicated by Table VIII is probably a result of the general density increase in the corona which accompanies a rise in the level of solar activity (see, for example, Elwert, 1963; De Jager, 1964). This possibility will be considered again in the next sub-section.

\section{E. SOFT X-RAY AMPLITUDE RELATED TO PLAGE CHARACTERISTICS}

If the general level of solar activity over the entire disk is related to the amplitude of soft X-ray bursts accompanying flares, as shown above for importance in flares, 
then it seems reasonable to expect that a similar relationship might also exist with the specific activity level of the burst-associated plage itself. In order to test this possibility, three indices of plage activity were considered: the flare-richness of the plage, its age, and the area of its major spot group.

The frequency distribution of the active regions as a function of their fiare-richness is shown in Figure 6, which indicates how many plages were responsible for a given number of events in Catalog II. The plages were then divided into those which produced more than 10 cataloged events and those which produced 5 or less, called 'prolific' and 'nonprolific' regions, respectively. The group of flare-producing regions we defined as 'prolific' consisted of McMath Nos. 8740, 8818, 8905, 8942 and 9184.

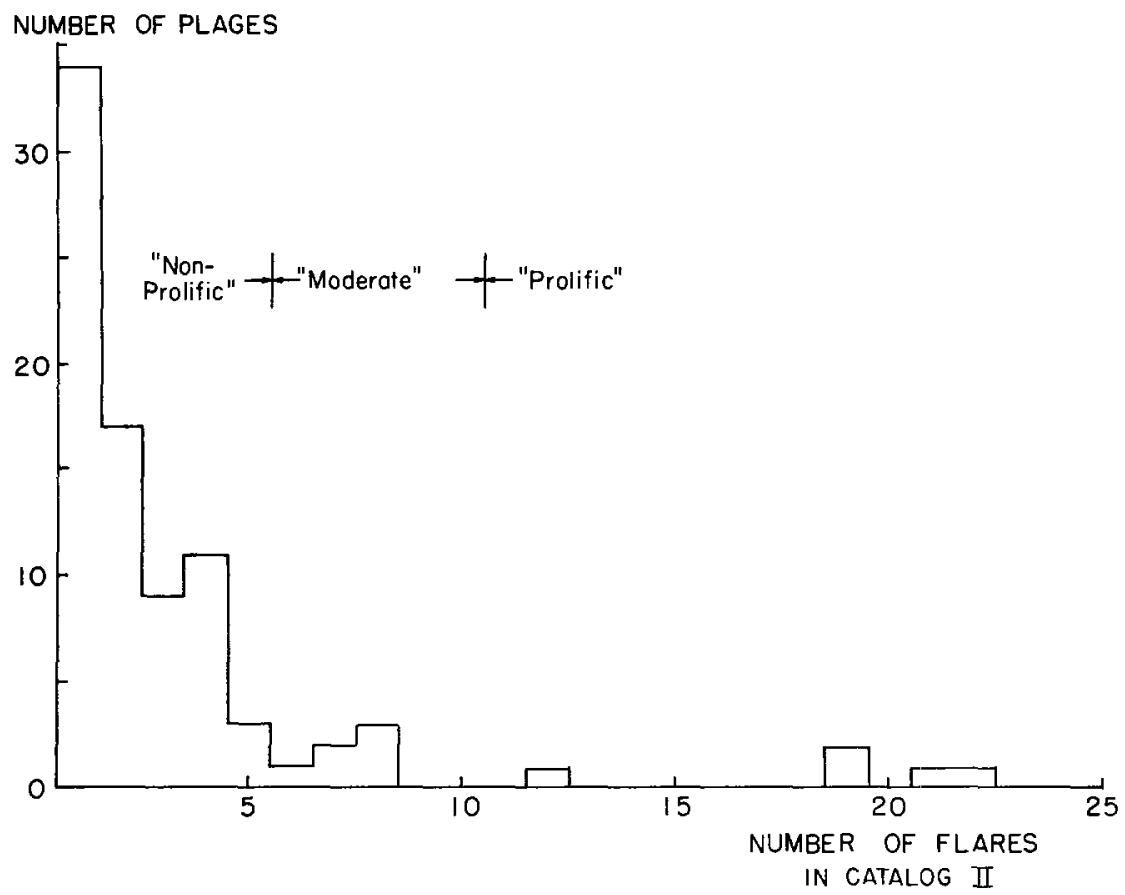

Fig. 6. Frequency distribution of plages having a given number of flares in Catalog II. Plages which produced more than $\mathbf{1 0}$ cataloged flares are considered 'prolific'. Those which produced 5 or less are defined as 'nonprolific'.

Plage regions McMath Nos. 8791, 8907, 9047, 9115, 9146 and 9204 were not included in either category, being considered as moderately flare-rich. This division is not exactly the same as the one made in Paper I because of the different flare catalogs used. The first two entries of Table IX show that the mean amplitude of bursts with importance 1n flares is significantly higher for those which are associated with prolific plage regions; there is only a $2 \%$ probability that this result could be due to chance. Such a result already has been suggested in Paper I by an investigation of median burst amplitudes, although the division of plages into prolific and nonprolific 
categories was somewhat different. Using the plage division given in Paper I, Culhane and Phillips (1970) have also found the same effect for 3-4 $\AA$ soft X-ray bursts.

The second parameter considered was the age of the plage as measured by the number of solar rotations it had been visible since its formation. All cataloged bursts were used which occurred in plages with a single age given by the SGDB. (Two plages may merge together forming a single active region; if the ages of the parent regions were different, the SGDB lists both for the resulting plage.) The bottom portion of Table IX shows that there is a steady decline in the mean X-ray burst amplitude with importance $\ln$ flares as the associated plage region becomes older. Unfortunately,

TABLE IX

Mean soft X-ray amplitude for importance $1 \mathrm{n}$ flares versus plage characteristics

\begin{tabular}{lll} 
Plage characteristic & $\begin{array}{l}\overline{\Delta E}(8,12) \\
\mathrm{erg} \mathrm{cm}^{-2} \mathrm{sec}^{-1}\end{array}$ \\
\hline Flare richness: prolific & $0.0244 \pm 0.0025$ p.e. \\
nonprolific & $0.0155 \pm 0.0014$ \\
1 & $0.0190 \pm 0.0023$ p.e. \\
2 & 0.01810 .0013 \\
3 & 0.0169 & 0.0021 \\
4 & 0.0165 & 0.0050 \\
5 & 0.0125 & 0.0031 \\
\hline
\end{tabular}

there were too few qualified events in these averages, especially for plage ages of 4 and 5 rotations, to make the result statistically conclusive, and a much more extensive set of X-ray burst observations will be necessary to confirm this finding. But the monotonic decrease in the mean amplitude values derived here is certainly very suggestive that a region's age is somehow related to its X-ray producing capability during a flare.

A physical basis for such a relationship has been reported by Neupert $(1967,1968)$, who found that the temperature and density of the condensation overlying a plage both decline as the region ages. All realistic thermal mechanisms for the production of soft $X$-radiation predict that the only physical conditions directly related to the $\mathrm{X}$-ray emission rate are the temperature and density, as well as volume, of the radiating source (Acton, 1964). Thus Neupert's finding is completely consistent with the result of the above study if these physical conditions normally undergo the same percentage change in the $\mathrm{X}$-ray source during an $\mathrm{H} \alpha$ flare of a given importance. This requirement also seems necessary to explain the relationships described in the previous section. Further support for this hypothesis is given by Švestka (1963), who finds that anomolously high X-ray enhancements accompanied importance 1 flares which occurred in a region with an abnormally high density according to one interpretation of its optical spectrum. 
It should be noted, however, that the two effects indicated in Table IX are not completely independent, since flare-rich plages are usually also young. Typically the flaring frequency within a region diminishes as the plage grows older (e.g. Giovanelli, 1939; Witte, 1951).

Another semi-independent index of a region's activity level is the area of its sunspots. Since a plage may sometimes contain two or more sunspot groups, we used here the total area of the group nearest the flare itself, as tabulated in the Rome Solar Phenomena Bulletin (Cimino, 1967). Whenever the identity of the proper spot group could not be established unambiguously, the event was eliminated from further consideration. Furthermore, this study examines only those bursts which accompanied flares of importance $1 \mathrm{n}$ or $\mathrm{lb}$ in McMath plage regions Nos. $8740,8818,8905$, or 8942 , and occurred within $72^{\circ}$ heliocentric of the solar disk center $(R \leqslant 0.95)$.

Figure 7 shows the resulting distribution of X-ray burst amplitude as a function of the related sunspot area. There seems to be no direct relationship between individual

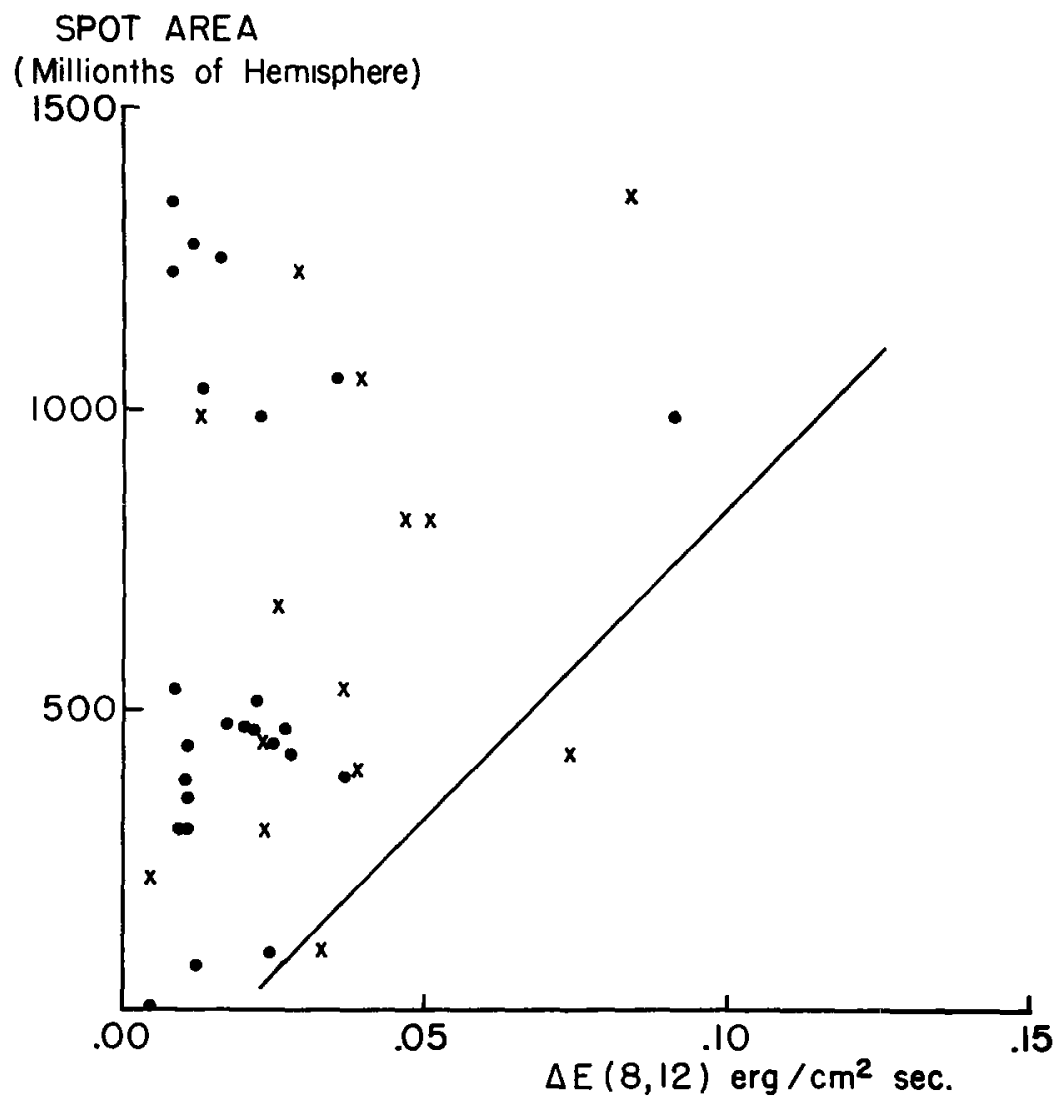

Fig. 7. Scatter diagram of $\Delta E(8,12)$ versus total area of the related sunspot group. X-ray bursts associated with importance in flares are shown as 0 ; those with $1 \mathrm{~b}$ flares as $\times$. Note the relative absence of points to the right of the line arbitrarily drawn in the figure. 
values. But the relative absence of points in the lower-right portion of the figure might be interpreted as implying that the region associated with a sunspot of given area can produce an X-ray burst of any amplitude up to a certain limiting value, and that the amplitude limit increases with the size of the spot group. This finding is consistent with the results of Dodson and Hedeman's (1970) examination of major flares (importance 2 or larger) which occur in plages with very small or no sunspots. They report that these flares were associated with a lower than normal percentage of both great SWF's and large amplitude 2-12 $\AA$ X-ray bursts. They further note that: "These flares usually occurred during the late, flare-poor phase of a center of activity", a statement which agrees precisely with two aspects of the present study described above.

These results can be explained if the X-ray emission rate were governed by the temperature and density of the source region as implied by the first two studies in this section, but confined to a range given by the region's magnetic energy density, which is crudely indicated by the area of the associated sunspot group. To test this suggestion properly, one should also consider the classification of the sunspot group's complexity and measured magnetic field strength, in addition to its total area, but this will be left for future investigation.

\section{F. SOFT X-RAY AMPLITUDE WITH FLARES HAVING TYPE IV RADIATION}

Tandberg-Hanssen (1967) has noted that flares which are most productive of Xradiation are also those that produce strong Type IV radio bursts. The present study indicates that at least some X-ray enhancements with accompanying Type IV do not follow that relation. Table $X$ lists the nine events in Catalog II which were associated with a Type IV radio burst reported in SGDB and which had X-ray coverage by OSO-III at the time of X-ray maximum. Type IV bursts are rare; they occurred with only 15 cases of the 283 flares in the Catalog. For six of these the maximum of the $\mathrm{X}$-ray enhancement was not observed.

We have found that the Catalog II flares associated with Type IV bursts are not invariably accompanied by outstanding X-ray bursts (see Table X). Except for the

TABLE $X$

Soft X-ray events associated with Type IV radio bursts

\begin{tabular}{|c|c|c|c|c|}
\hline Date & $\begin{array}{l}\mathrm{H} \alpha \text { fiare } \\
\text { importance }\end{array}$ & $\begin{array}{l}\text { Type IV burst } \\
\text { importance }\end{array}$ & $\begin{array}{l}\Delta E(8,12) \\
\mathrm{erg} \mathrm{cm}^{-2} \mathrm{sec}^{-1}\end{array}$ & $\Delta E / \overline{\Delta E}$ \\
\hline 22 March, 1967 & $2 b$ & 2 & 0.1211 & 1.2 \\
\hline 23 March, 1967 & $1 b$ & 2 & 0.0257 & 0.7 \\
\hline 21 May, 1967 & $2 n$ & 3 & 0.2635 & 6.2 \\
\hline 23 May, 1967 & $3 b$ & 3 & 0.8060 & - \\
\hline 26 August, 1967 & $1 b$ & 3 & 0.0460 & 1.3 \\
\hline 18 September, 1967 & $2 b$ & 3 & 0.0400 & 0.4 \\
\hline 25 October, 1967 & $\ln$ & 3 & 0.0083 & 0.5 \\
\hline 11 January, 1968 & $1 b$ & 2 & 0.0643 & 1.9 \\
\hline 10 February, 1968 & $2 \mathrm{n}$ & 3 & 0.0319 & 0.8 \\
\hline
\end{tabular}


flares of 21 and 23 May, 1967, the X-ray amplitudes do not appear to be exceptional. To show this we have calculated the ratio of the observed X-ray amplitude to the mean amplitude for the appropriate flare importances which are given in Table III. The ratio was not calculated for the 23 May, 1967 flare since it is the only example of its importance class in the Catalog. Of the eight remaining events, only four had soft $\mathrm{X}$-ray amplitudes larger than the mean value for their importance class. Although our sample is too small to be definitive, it suggests that Type IV radiation is not restricted solely to flares having exceptional soft X-ray enhancements.

\section{G. DEPENDENCE OF SOFT X-RAY AMPLITUDE UPON CM- $\lambda$ COMPONENT OF RADIO SPECTRUM}

We investigated the radio burst spectrum for all flares in Catalog II for which we had X-ray amplitude data. Radio spectra were estimated from the reported singlefrequency flux-density enhancements at maximum without regard to small timedifferences in the times of single-frequency maxima, so long as these differences did not exceed several minutes. The radio burst spectra were divided into two groups: Class A, characterized by spectra having the greatest reported flux at the highest frequency listed, normally about $10000 \mathrm{MHz}$; and Class B, with spectra having the greatest reported flux at a lower frequency.

Since a particularly complete set of radio observations is necessary to categorize these bursts properly, only 54 events were found with clearly defined spectral type and for which an X-ray burst amplitude was listed in Catalog II.

TABLE XI

Mean soft X-ray amplitude versus spectrum of radio burst

\begin{tabular}{llll}
\hline & \multicolumn{4}{c}{$\overline{\Delta E}(8,12) \mathrm{erg} \mathrm{cm}^{-2} \mathrm{sec}^{-1}$} & \\
\cline { 2 - 4 } & \multicolumn{1}{c}{ Spectral class 'A' } & Spectral class 'B' & $P$ \\
\hline All events & $0.0983 \pm 0.0293$ p.e. & $0.0308 \pm 0.0049$ p.e. & $96 \%$ \\
'In' events & $0.0526 \pm 0.0191$ & $0.0199 \pm 0.0018$ & $94 \%$ \\
\hline
\end{tabular}

The first line of Table XI shows that the mean amplitude for all soft X-ray bursts accompanying Class $A$ radio events is higher than that for Class $B$, with a $96 \%$ probability that this effect is real. But there is a tendency for the Class A bursts to be associated with the very largest $\mathrm{H} \alpha$ flares; $61 \%$ of these bursts occurred during flares of importance $1 \mathrm{~b}$ or greater, compared to only $44 \%$ of the Class B bursts. Thus, it is conceivable that the higher mean amplitude of X-ray bursts accompanying Class $A$ radio events is accounted for by the relation found in Section $3 B$ between $\overrightarrow{\Delta E}(8,12)$ and $\mathrm{H} \alpha$ flare importance. The second line of Table XI shows that this is not the case, however. Here only events which occurred during importance in flares were considered. Yet the mean X-ray amplitude still is higher for bursts accompanied by Class $A$ radio events, with just a $6 \%$ probability that this observed difference is due to chance. A similar result for hard X-ray bursts can be inferred from the report by Arnoldy et al. (1968), that the probability of detecting a $0.2-1.2 \AA$ X-ray event is 
negligible unless the spectrum of the associated radio burst includes $\mathrm{cm}-\lambda$ enhancements.

Unfortunately, the interpretation of a spectrum obtained from the peak fluxes of single-frequency data is by no means straightforward (cf. Kundu, 1962), so that the physical meaning of the relationship found above is not clear. Furthermore, there is a possibility that the sample of events studied here was somehow biased. Kundu (1965) finds that the peak flux of a cm- $\lambda$ radio burst usually increases with increasing frequency, while only $33 \%$ of the events in the present investigation showed that property. In any case, the relation indicated here between the X-ray burst amplitude and the spectral type of the associated radio burst needs to be confirmed by a more refined analysis.

\section{Total Energies in Soft X-Ray Bursts}

Time-integrals of soft X-ray bursts in the range 8-12 $\AA$ have been determined by one of us (Teske, 1969) for subflares and flares of importance 1. In the present work we have extended the estimation of time-integrated soft X-ray energies to flares of all importance classes found in our Catalog II. Intercomparison of the results is meaningful insofar as they are independent of the spectral distribution to within $30 \%$, although the quoted total energies may be too high by a factor of two or three (see above).

TABLE XII

Total 8-12 Å emission during $\mathrm{H} x$ flares

\begin{tabular}{ll}
\hline $\begin{array}{l}\text { H } \alpha \text { flare } \\
\text { importance }\end{array}$ & $\begin{array}{l}\text { Mean total 8-12 } \\
\text { emission (erg) }\end{array}$ \\
\hline 1f & $2 \times 10^{28}$ \\
1n & $5 \times 10^{28}$ \\
1b & $1 \times 10^{29}$ \\
2f & $1 \times 10^{29}$ \\
2n & $2 \times 10^{29}$ \\
2b & $5 \times 10^{29}$ \\
3n & $1 \times 10^{30}$ \\
3b & $1 \times 10^{31}$ \\
\hline
\end{tabular}

Table XII lists the time-integrated soft X-ray energies which were estimated by approximating the profile of the X-ray burst by a triangle. We used as amplitude for each importance class the mean amplitude given in. Table III and took 2000, 4000 and $8000 \mathrm{sec}$ as the time-duration for flares of importance 1, 2 and 3, respectively. These $\mathrm{X}$-ray energies are compared in Figure 8 with the total $\mathrm{H} \alpha$ emission, $\int \Delta S(\mathrm{H} \alpha) \mathrm{d} t$, determined by Thomas (1970b) for corresponding flare importances. As in Figure 5, values are included for subflares that were measured for an earlier catalog. The straight line represents a linear relation between the two emitted energies. Since the measured points fit that relation fairly well, we have on the average:

$$
\int \Delta S(8,12) \mathrm{d} t=0.8 \int \Delta S(\mathrm{H} \alpha) \mathrm{d} t
$$




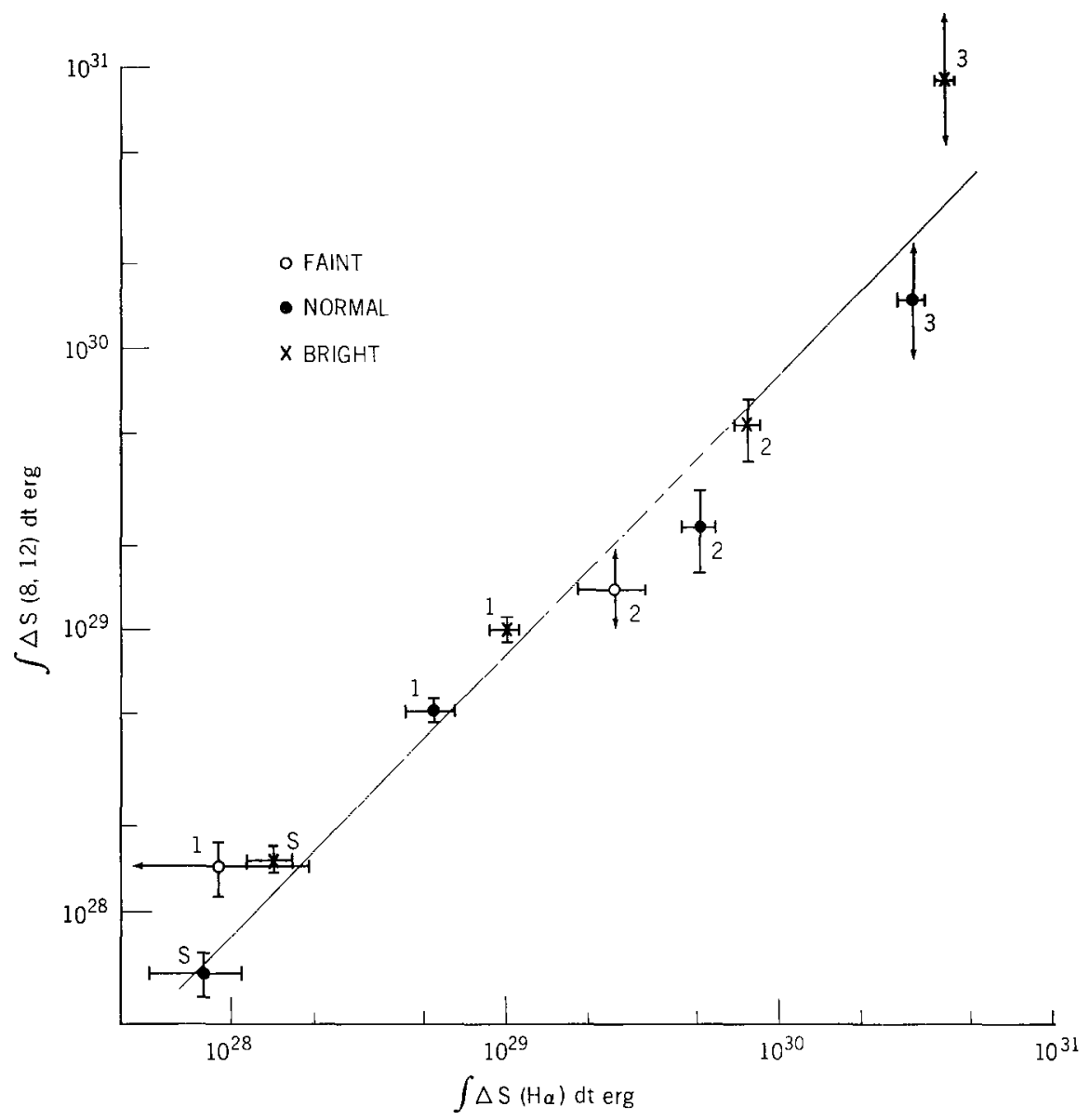

Fig. 8. Relation between total enhanced emission of 8-12 $\AA$ and $H \alpha$ radiation during flares of various importances. Error bars refer to the probable errors involved, except that no error can be assigned to $\int \Delta S(8,12) \mathrm{d} t$ associated with $2 \mathrm{f}, 3 \mathrm{n}$, or $3 \mathrm{~b}$ flares, since only one example of each of these bursts has been measured. The straight line indicates the best fit by a linear relationship (i.e., unity slope).

The time-integrated enhancements in the $\mathrm{H} \alpha$ and soft $\mathrm{X}$-ray emissions are therefore not only proportional to one another, they are very nearly the same. Even considering the errors in the absolute calibrations of these measurements, the total flare emissions in soft $\mathrm{X}$-ray and $\mathrm{H} \alpha$ radiations must be equal within an order of magnitude. Furthermore, the amount of energy emitted as soft X-radiation accounts for a significant portion of the total electromagnetic energy lost during a flare event. A comparison of the values in Table XII with reported values for the total flare emission at other wavelengths (see, for example, Thomas, 1970a) shows that this fraction may be as high as $10 \%$ for flares in general. 


\section{Summary}

Virtually all solar flares are accompanied by enhanced soft $X$-radiation which is predominantly thermal in nature. Although each flare is unique, there are some characteristics of the associated soft $X$-ray burst that we consider as 'typical'. On the average, the burst begins about two minutes before the start of the $\mathrm{H} \alpha$ event, reaches its peak flux about three minutes after the maximum $\mathrm{H} \alpha$ intensity, and ends several minutes after the optical flare is no longer visible. For those $\mathrm{X}$-ray events observed with high flux sensitivity, the initial rate of rise is very gradual and often becomes more rapid at the onset of the $\mathrm{H} \alpha$ flare. In general, the soft $\mathrm{X}$-ray flux and the intensity of the $\mathrm{H} \alpha$ flare's brightest point have roughly similar time-profiles.

The strength of the X-ray burst is directly related to both the size and the brilliance of the flare with which it occurs. Indeed, the peak enhancements in the soft X-ray and $\mathrm{H} x$ emission rates during flares are of the same order of magnitude, as are the total flare energies radiated at these wavelengths. We estimate that soft X-radiation accounts for up to one-tenth of a flare's total electromagnetic emission.

The X-ray burst's strength also depends on the general level of solar activity at the time of the event and on the age and 'flare-richness' of the associated plage, effects which are most likely due to density variations in the $\mathrm{X}$-ray emitting region itself. In addition, those flares with radio bursts which are dominated by $\mathrm{cm}-\lambda$ emission tend to produce particularly strong soft X-ray enhancements.

There is some evidence that certain relationships between the $\mathrm{H} \alpha$ and $\mathrm{X}$-ray emission during flares vary with the distance of the event from the solar limb. This would imply a variation of the optical flare's characteristics with height, since only the higher levels of the $\mathrm{H} \alpha$ event are visible close to the limb while no such effect is expected for the associated soft X-ray enhancement.

\section{Acknowledgement}

This work has been sponsored in part by Contract NAS5-3176 with the National Aeronautics and Space Administration.

\section{References}

Acton, L. W.: 1964, Doctoral Dissertation, University of Colorado.

Acton, L. W., Chubb, T. A., Kreplin, R. W., and Meekins, J. F.: 1963, J. Geophys. Res. 68, 3335. Angle, K. L.: 1968, Astron. J. 73, S53 (Abstract).

Arnoldy, R. L., Kane, S. R., and Winckler, J. R.: 1968, Astrophys. J. 151, 711.

Chambe, G. and Sain, M.: 1969, Astron. Astrophys. 2, 133.

Cimino, M.: 1967, Solar Phenomena, Osservatorio Astronomico di Roma, No. 111-116.

Covington, A. E.: 1959, in Paris Symposium on Radio Astronomy (ed. by R. N. Bracewell), Stanford University Press, p. 159.

Covington, A. E. and Harvey, G. A.: 1958, J. Roy. Astron. Soc. Can. 52, 161.

Culhane, J. L.: Personal Communication.

Culhane, J. L. and Phillips, K. J. H.: 1970, Solar Phys. 11, 117.

Culhane, J. L., Willmore, A. P., Pounds, K. A., and Sanford, P. W.: 1963, Space Res. 4, 741. 
De Jager, C.: 1964, Research in Geophysics Vol, 1, M.I.T. Press, Cambridge, Mass.

De Jager, C.: 1965, Ann. Astrophys. 28, 125.

Dodson, H. W. and Hedeman, E. R.: 1960, J. Geophys. Res. 65, 123.

Dodson, H. W. and Hedeman, E. R.: 1964, Planetary Space Sci. 12, 393.

Dodson, H. W. and Hedeman, E. R.: 1970, Solar Phys, 13, 401.

Dodson, H. W., Hedeman, E. R., and McMath, R. R.: 1965, Astrophys. J. Suppl. 2 , 241.

Donnelly, R. F.: 1968, 'Early Detection of a Solar Flare: A Study of X-Ray, Extreme Ultraviolet, $\mathrm{H} \alpha$, and Solar Radio Emission from Solar Flares', ESSA Technical Report ERL 81-SDL 2, July.

Drake, J. F.: Personal Communication.

Drake, J. F.: 1969, 'Characteristics of 2-12 $\AA$ Solar X-Ray Flares', University of Iowa Report No. 69-41, August.

Elwert, G.: 1963, Proc. Intern. Conf. Ionosphere, July 1962, p. 57.

Falciani, R., Landini, M., Righini, A., and Rigutti, M.: 1968, in K. O. Kiepenheuer (ed.), 'Structure and Development of Solar Active Regions', I.A.U. Symp. 35, 451.

Giovanelli, R. G.: 1938, Astrophys. J. 88, 204.

Giovanelli, R. G.: 1939, Astrophys. J. 89, 555.

Gregory, B. N. and Kreplin, R. W.: 1967, J. Geophys. Res. 72, 4815.

Holt, S. S. and Cline, T. L.: 1968, Astrophys. J. 154, 1027.

Hudson, H. S., Peterson, L. E., and Schwartz, D. A.: 1969, Astrophys. J. 157, 389.

Kane, S. R.: 1969, Astrophys. J. 157, L139.

Kawabata, K.: 1963, in J. W. Evans (ed.), 'The Solar Corona', I.A.U. Symp. 16, 143.

Kawabata, K.: 1966, Rep. Ionosphere Space Res. Japan $20,118$.

Kundu, M. R.: 1962, J. Geophys. Res. 67, 2695.

Kundu, M. R.: 1964, in AAS-NASA Symposium on the Physics of Solar Flares (ed. by W. N. Hess), NASA SP-50, p. 335.

Kundu, M. R.: 1965, Solar Radio Astronomy, Interscience Press, New York.

Landini, M., Russo, D., and Tagliaferri, G. L.: 1965, Space Res. 6, 1041.

Lindsay, J. C.: 1964, Planetary Space Sci. 12, 379.

Neupert, W. M.: 1967, Solar Phys. 2, 294.

Neupert, W. M.: 1968, in K. O. Kiepenheuer (ed.), 'Structure and Development of Solar Active Regions', I.A.U. Symp. 35, 404.

Paolini, F. R., Giacconi, R., Manley, O., Reidy, W. P., Vaiana, G. S., and Zehnpfennig, T.: 1968, Astron. J. 73, S73 (Abstract).

Reid, J. H.: 1969, J. Atmospheric Terrest. Phys. 31, 859.

Sawyer, C.: 1967, Astrophys. J. 147, 1135.

Smith, H. J.: 1962, 'Some Synoptic Flare Data, 1937-1960', GRD Research Note, AFCRL-62-827.

Švestka, Z.: 1963, Space Res. 4, 768.

Takakura, T. and Kai, K.: 1966, Publ. Astron. Soc. Japan 18, 57.

Tandberg-Hanssen, E.: 1967, Solar Activity, Blaisdell Press, Waltham. Mass.

Teske, R. G.: 1967, Astron. J. 72, 832 (Abstract).

Teske, R. G.: 1969, Solar Phys. 6, 193.

Teske, R. G. and Thomas, R. J.: 1969, Solar Phys. 8, 348.

Thomas, R. J.: 1970a, Doctoral Dissertation, University of Michigan.

Thomas, R. J.: 1970b, Submitted to Solar Phys.

Waldmeier, M. and Bachmann, H.: 1959, Z. Astrophys. 47, 81.

Warwick, C. S.: 1963, in Radio Astronomical and Satellite Studies of the Atmosphere, (ed. by J. Aarons), North-Holland Publ. Co., Amsterdam, p. 457.

Warwick, C. S.: 1965, Astrophys. J. 142, 767.

Wende, C. D.: 1969, J. Geophys. Res. 74, 4649.

Witte, B.: 1951, 'A Contribution to the Study of the Relation between Solar Flares and Sunspot Groups', HAO Solar Research Memorandum. 